\title{
Restructuration du déversoir de Djorf-Torba
}

\section{Restructuring of the spillway of the Djorf-Torba dam}

\author{
M.S. Belouni et T. Sahraoui \\ Direction Générale de l'Exploitation, Ministère de l'Hydraulique, Alger
}

J. Bouloc

Laboratoire Central d'Hydraulique de France, Maisons-Alfort

B. Goguel

Bureau d'Ingénieurs-Conseils Coyne et Bellier, Paris

\section{Introduction}

Parallèlement au vaste programme de construction de barrages en cours de réalisation en Algérie, le Ministère de l'Hydraulique se préoccupe de la sécurité des ouvrages existants. Ainsi est apparu la nécessité de revoir l'évacuateur de crues du barrage de Djorf-Torba, achevé. en 1968 sur l'Oued Guir où il crée la plus grande retenue d'Algérie, malgré sa hauteur modeste de 35 mètres.

La partie centrale de ce long barrage-poids en béton est équipée de seuils déversants, pouvant lâcher jusqu'à $3500 \mathrm{~m}^{3} / \mathrm{s}$ sur un tapis déflecteur large de 100 mètres. Le fait que les seuils se présentent à deux niveaux différents interdisait d'aller très loin dans l'étude théorique de la restitution. Bien que le projet initial ait fait, à l'époque, l'objet d'études sur modèle réduit, la construction d'un nouveau modèle réduit hydraulique s'imposait, ne serait-ce que pour permettre l'introduction de données nouvelles apparues entre temps.

La construction de ce modèle, à l'échelle $1 / 50^{\mathrm{e}}$, et quelques mois d'essais ont permis de confirmer les craintes relatives à la stabilité de l'ouvrage dans son état actuel, mais aussi de réduire au minimum l'ampleur et la difficulté des travaux de consolidation nécessaires.

\section{Présentation de l'ouvrage}

\subsection{Description d'ensemble}

Le barrage poids a un profil classique, une grande longueur (762 mètres) et une implantation légèrement courbe ( $R=490$ mètres).

Le site présente une remarquable régularité structurale, avec des bancs calcaires subhorizontaux plongeant de quelques pour cent de la rive gauche vers la rive droite. Certains niveaux du Turonien supérieur apparaissent karstifiés dans les rives : en état de décomposition avancée en rive gauche, de façon plus franche en rive droite (où se posent des problèmes de contournement au large du voile d'injection).

La partie centrale déversante, où le barrage atteint sa plus grande hauteur ( 35 mètres), est fondée sur un calcaire marneux, légèrement fissuré, mais sain. Le lit de l'Oued, large d'une centaine de mètres, est constitué de galets, centimétriques à décimétriques, sur une demidouzaine de mètres d'épaisseur.

L'évacuateur de crues comporte deux passes centrales de $15 \mathrm{~m}$ de large chacune, calées à la côte 699 , c'est-à-dire à $7 \mathrm{~m}$ sous la crête du barrage et encadrées par deux fois trois passes latérales de $12 \mathrm{~m}$ de large, calées $2,5 \mathrm{~m}$ plus haut (Fig. 1). Les seuils sont libres, de profils classiques.

Les débits déversés sont restitués sur un radier horizontal, calé à la côte 673 (26 m sous le premier seuil), et des chute-blocs situés dans la zone de raccordement coursier-radier assurent une certaine dissipation d'énergie. Le tapis se termine abruptement au bout de 35 mètres avec un parafouille aval profond de 5 mëtres (Fig. 2).

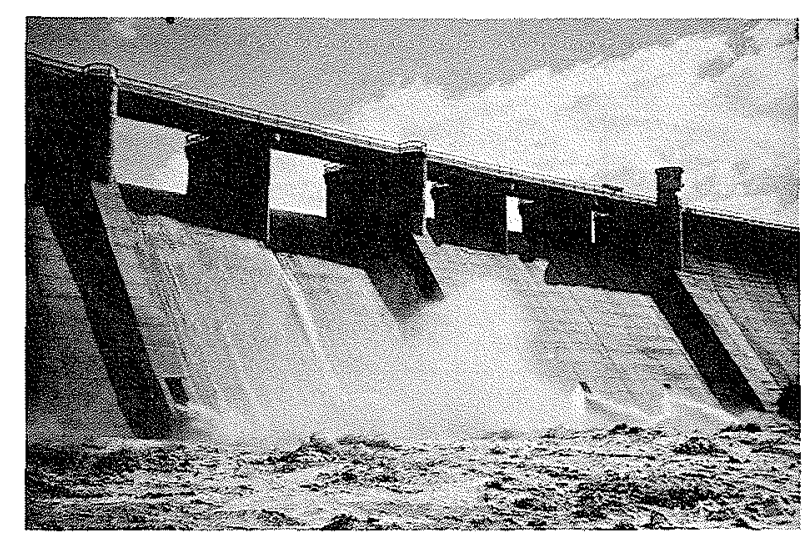

Figure 1 - Vue d'ensemble du déversoir de Djorf-Torba (photo prise le 22 octobre 1979, au débit maximal enregistré $: 305$ $\mathrm{m}^{3} / \mathrm{s}$ ). 


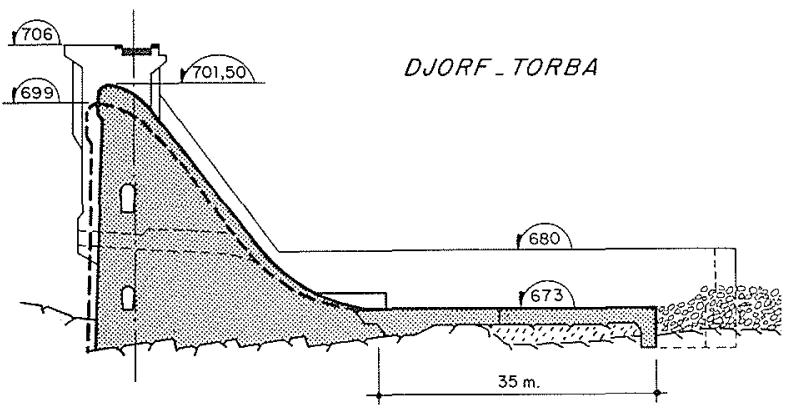

Figure 2 - Coupe sur déversoir.

L'ensemble s'étend sur une largeur d'une centaine de mètres dans l'axe de l'oued. Les deux murs-guideaux intermédiaires qui séparent les coursiers légèrement décalés ne se prolongent pas sur le tapis.

Sept pertuis de vidange de fond, pouvant débiter $30 \mathrm{~m}^{3} / \mathrm{s}$ chacun, sont installés dans l'axe des piles qui portent le pont routier au-dessus du déversoir.

\subsection{Hydrologie}

L'ouvrage contrôle un vaste bassin versant subsaharien, de superficie avoisinant $22000 \mathrm{~km}^{2}$, en tête de vallée de la Saoura. Il est destiné à amortir les crues violentes de l'oued Guir et à en régulariser les apports au profit des irrigations dans l'immense et fertile plaine d'Abadla, où les hommes luttent contre le désert. Une adduction vers Béchar sera en outre prochainement mise en service.

L'apport du Guir est très variable selon les années. D'après un bilan dressé après onze années d'exploitation, il avoisine 200 millions de mètres cubes d'eau par an en moyenne, ce qui est peu différent des premières évaluations qui avaient précédé la construction du barrage.

Mais des nouveautés sont apparues, par rapport aux anciennes évaluations, en ce qui concerne la statistique des crues. En deux ans, le chantier a vu passer deux crues dites décennales et une crue plus que centennale $\left(Q p=6000 \mathrm{~m}^{3} / \mathrm{s}, A=360 \mathrm{hm}^{3}\right.$, le 17 novembre 1967). D'autres crues d'apports bien connus se chiffrant en centaines de millions de mètres cubes ont été enregistrées ensuite par l'exploitation du barrage.

Il en est résulté une révision des estimations mentionnées dans le tableau I.

\begin{tabular}{|c|c|c|c|c|}
\hline \multirow{2}{*}{$\begin{array}{c}\text { Période de } \\
\text { retour } \\
\text { (années) }\end{array}$} & \multicolumn{2}{|c|}{ Anciennes } & \multicolumn{2}{c|}{ Nouvelles } \\
\cline { 2 - 5 } & $\begin{array}{c}O p \\
\left(\mathrm{~m}^{3} / \mathrm{s}\right)\end{array}$ & $\begin{array}{c}A \\
\left.\mathrm{hm}^{3}\right)\end{array}$ & $\begin{array}{c}O P \\
\left(\mathrm{~m}^{3} / \mathrm{s}\right)\end{array}$ & $\begin{array}{c}A \\
\left(\mathrm{hm}^{3}\right)\end{array}$ \\
\hline 10 & 2500 & 107 & 4400 & 240 \\
100 & 5000 & 350 & 8800 & 485 \\
1000 & 8000 & 760 & 15000 & 820 \\
\hline
\end{tabular}

La révision a surtout porté sur les crues relativement courantes, directement observées : le volume de la crue décennale a plus que doublé, la crue centennale est devenue vingt à trentennale.

Cette nécessaire inflation n'a pas été poussée aux apports de fréquences exceptionnelles, faute de données suffisantes. Mais le développement des connaissances relatives à l'hydrologie des bordures sahariennes rend plausibles des "crues de projet" dont l'apport dépasserait sensiblement le milliard de mètres cubes. On connait par exemple le cas de l'Oued Zeroud à Sidi-Saad en Tunisie, à l'ouest de Kairouan : bassin versant d'environ $9000 \mathrm{~km}^{2}$, apport moyen annuel de l'ordre de $100 \mathrm{hm}^{3}$, deux caractéristiques moitiés de celles du Guir à DjorfTorba, avec un régime climatique assez comparable. Lors de la plus forte des quatres crues de l'automne 1969, cet Oued a roulé plus d'un milliard de mètres cubes d'apport en deux jours avec une pointe jaugée à plus de 15000 $\mathrm{m}^{3} / \mathrm{s}$.

\subsection{Retenue et amortissement des crues}

La tranche pratique d'exploitation de la retenue est limitée à la cote 697 , et représente $250 \mathrm{hm}^{3}$ de capacité.

S'y ajoutent pour l'amortissement des crues :

$100 \mathrm{hm}^{3}$ sous le premier seuil déversant (cote 699 )

$+550 \mathrm{hm}^{3}$ entre ce premier seuil et le niveau maximal théorique (cote $706=$ origine du profil triangulaire du barrage-poids et route de crête).

La superficie de la retenue est de 4600 hectares à la cote 697 , et de 10500 ha à la cote 706 .

Le creux minimal de $100 \mathrm{hm}^{3}$ sous le déversoir a pour but de réduire les dégâts causés à l'aval par les crues relativement courantes. Telle est également la raison du double niveau de seuils déversants.

Le niveau 697 jusqu'à présent n’a été dépassé que trois fois (Fig. 3), avec déversements en 1975 et, au maximum, le 22 octobre 1979 : cote atteinte 700,62 , débits évacués $105 \mathrm{~m}^{3} / \mathrm{s}$ par la surface et $200 \mathrm{~m}^{3} / \mathrm{s}$ par les vidanges de fond (Fig. 1).

L'importance du laminage est considérable pour la protection de l'ouvrage contre les crues. Les crues courantes déversent rarement. Les crues plus rares sont temporairement stockées pour 80 à $90 \%$ de leur volume, le débit maximal déversé n'atteignant que 10 à $20 \%$ du débit de pointe entrant dans la retenue. Parmi les renseignements hydrologiques utiles à l'appréciation des risques, le débit de pointe n'a plus aucune importance : seul compte le volume d'apport concentré en quelques dizaines d'heures, et le débit de base qui pourrait le précéder et suffire à remplir la retenue, réduisant ainsi les possibilités d'amortissement.

La révision des estimations hydrologiques relatives aux crues a entraîné une révision des risques de déversement, en hausse par rapport aux estimations des constructeurs. On estime, aujourd'hui, qu'il suffirait d'une crue cinquante à centennale, au lieu de millénale, pour que l'ouvrage déverse $1000 \mathrm{~m}^{3} / \mathrm{s}$. Le niveau des PHE

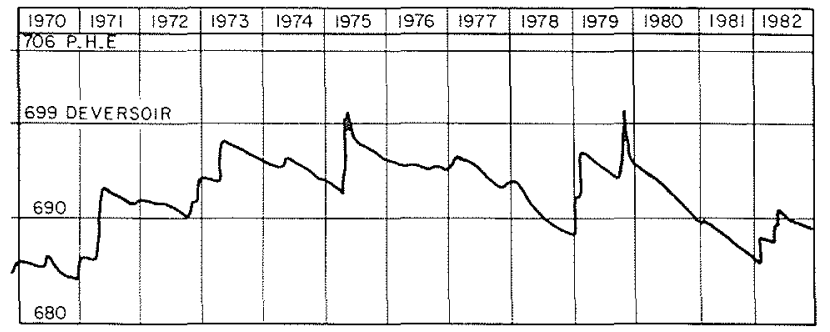

Figure 3 - Historique de la mise en eau de Djorf-Torba. 


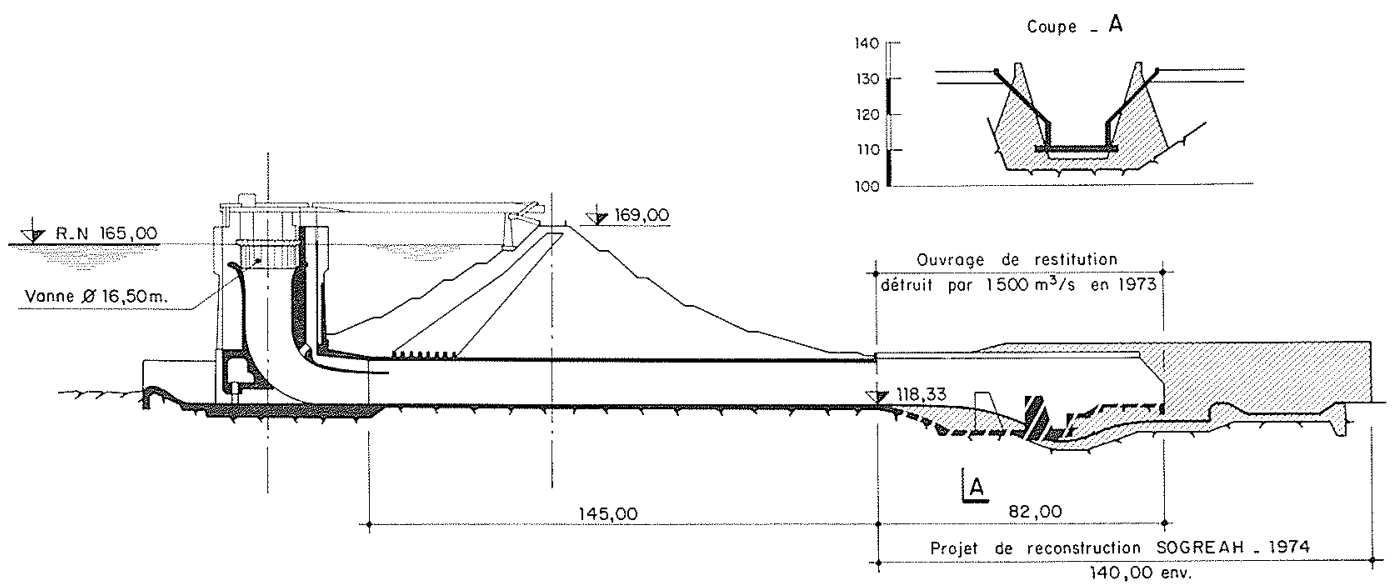

Figure 4 - Evacuateur de la Chéffia.

théoriques 706 , sous lequel déversent $2700 \mathrm{~m}^{3} / \mathrm{s}$, est affecté d'un risque cinqcentennal. Faute de pouvoir envisager plus, on estime enfin devoir prendre en considération des crues remplissant la retenue jusqu'à 706,85 (le parapet dépasse encore de 60 centimètres), soit $3500 \mathrm{~m}^{3} / \mathrm{s}$ à l'extrême sur le déversoir.

\subsection{Exploitation}

Le bilan dressé au terme de la période d'exploitation 1970-1982 montre que les $200 \mathrm{hm}^{3}$ d'apport moyen annuel se sont répartis presque à égalité entre trois destinations:

- un tiers a été évaporé dans la retenue (à raison de 2,3 mètres par an),

- un tiers a été déversé ou lâché par les vidanges pour revenir à la cote 697 (en mars, puis avril 1975, et octobre 1979),

- un tiers a été lâché dans l'Oued (à raison de 1,5 à $3,5 \mathrm{~m}^{3} / \mathrm{s}$, dont $20 \%$ par les fuites de la rive droite et le reste modulé par l'une des vidanges de fond). La reprise s'effectue $58 \mathrm{~km}$ plus loin, en tête des périmètres d'Abadla.

Les sept vidanges de fond servent à la fois au contrôle du niveau de la retenue, en permettant de débiter jusqu'à $200 \mathrm{~m}^{3} / \mathrm{s}$ au total dans le bassin de restitution du déversoir, à la lutte contre l'envasement à l'occasion de ces chasses, et à la modulation des lâchures d'irrigation.

L'absence de pertuis spécialisé indépendant de l'évacuateur de crues est à signaler : cette absence entraîne de lourdes sujétions pour inspecter le tapis du déversoir, ou y travailler, du fait que la continuité des lâchures est vitale pour les activités qui se sont développées à l'aval du barrage. Une conduite branchée sur le premier pertuis de vidange et sortant de l'emprise du tapis a été installée en 1982 pour pallier cet inconvénient.

\section{Craintes, et mesures de consolidation primaire}

\subsection{Naissance des doutes}

Le barrage a bel aspect, et le déversoir s'était fort bien comporté lors des déversements (minimes) survenus en
1975. Mais les abondantes percolations dans les rives karstifiées avaient attiré l'attention du Ministère de l'Hydraulique au point qu'il commanda quelques années plus tard une expertise d'ensemble de la stabilité du barrage.

La consultation des plans d'exécution, fort clairs et détaillés, montra qu'à l'évidence les concepteurs, à l'époque des travaux, ne s'étaient pas suffisamment souciés de certaines dispositions, reconnues aujourd'hui critiques pour la stabilité d'un grand ouvrage hydraulique.

Ces constatations coïncidaient avec la révision des estimations hydrologiques exposée précédemment : on n'avait plus à traiter des débits de quelques centaines de $\mathrm{m}^{3} / \mathrm{s}$, comme avaient pu le croire les constructeurs, mais à envisager le passage de plusieurs milliers de $\mathrm{m}^{3} / \mathrm{s}$, lié à une excursion plausible de la retenue à des cotes élevées.

Un précédent marquant donnait du poids aux craintes relatives à la stabilité du tapis du déversoir : la destruction accidentelle du bassin de dissipation de la Cheffia le 27 mars 1973. Construit sur l'Oued Bou-Namoussa de 1960 à 1964 , ce bassin prévu pour $2000 \mathrm{~m}^{3} / \mathrm{s}$ n'a pas résisté à une ouverture de la vanne d'évacuation qui a lâché $1500 \mathrm{~m}^{3} / \mathrm{s}$ (Fig. 4).

L'expertise des dégâts survenus depuis 1974 aux ouvrages de restitution de Tarbela au Pakistan montrait aussi combien un grand bassin à ressaut peut se révéler fragile.

\subsection{Conditions hydrauliques : bassin à ressaut, ou tapis déflecteur}

Les eaux dévalent de plus de 30 mètres et atteignent des vitesses proches de $25 \mathrm{~m} / \mathrm{s}$. Le radier horizontal, qui s'étend jusqu'à $35 \mathrm{~m}$ à l'aval des plots déversants du barrage, apparaît un peu court et calé trop haut, compte tenu du niveau aval, pour assurer une bonne tranquillisation.

La relation "hauteur-débit" dans l'Oued à l'aval est bien connue; elle a pu être vérifiée jusqu'à $5000 \mathrm{~m}^{3} / \mathrm{s}$ lors du passage de la crue de 1967 (légèrement amortie par le barrage en construction).

L'étude théorique bidimensionnelle des écoulements dans les passes centrales et latérales (Fig. 5) montre que 


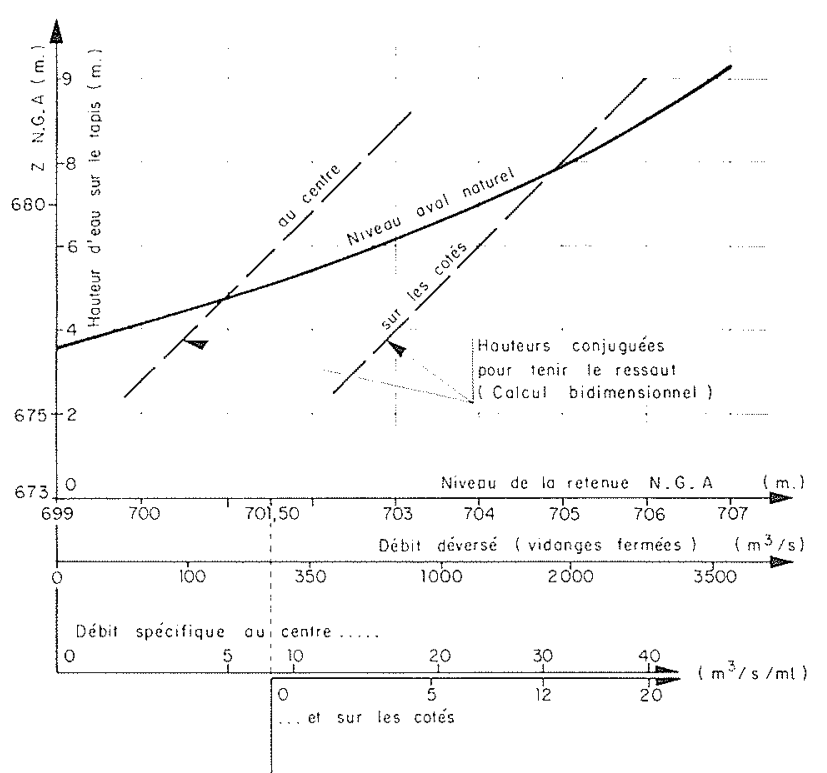

Figure 5 - Conditions hydrauliques.

la hauteur conjuguée nécessaire pour tenir le ressaut n'est plus assurée par le niveau aval :

- au centre, dès que l'évacuateur débite $260 \mathrm{~m}^{3} / \mathrm{s}$ (c'est-à-dire pour un niveau de retenue dépassant à peine la cote 701,50 de calage des seuils latéraux),

- sur les côtés, lorsque l'évacuateur débite $2000 \mathrm{~m}^{3} / \mathrm{s}$ (nivealı amont avoisinant 705 , risque centennal à cinqcentennal).

Pour tenir le ressaut sur le tapis jusqu'à $3000 \mathrm{~m}^{3} / \mathrm{s}$ avec le niveau aval naturel, il aurait fallu caler le tapis 10 mètres plus bas au centre, et 5 mètres plus bas sur les côtés.

Les constructeurs supposaient pouvoir bénéficier aux petits débits d'un effet tridimensionnel sur le tapis. Ils escomptaient aussi que le tapis serait naturellement prolongé par un seuil rocheux aval, seuil qui n'a en fait pas été retrouvé sous les alluvions.

Ils n'ont cependant pas monté les bajoyers au-delà de la cote 680 , soit 7 mètres au-dessus du tapis, ce qui correspond au niveau naturel dans l'oued pour 1000 $\mathrm{m}^{3} / \mathrm{s}$. Mais les $3500 \mathrm{~m}^{3} / \mathrm{s}$ passent à près de 3 mètres au-dessus de cette cote.

\subsection{Dispositions constructives}

Seuls les chute-blocs à l'amont du tapis, larges et espacés de $2 \mathrm{~m}$ et hauts de $2,50 \mathrm{~m}$, sont ferraillés.

Le radier horizontal, à la cote 673 , a une épaisseur de 2 mètres en partie courante et est constitué de deux bétons différents (70/300 sur les $50 \mathrm{~cm}$ supérieurs, $150 / 250$ en dessous). Il n'est ni armé, ni ancré.

Il n'existe aucun waterstop ni dispositif d'étanchéité équivalent sur les joints de construction, disposés dans les deux sens tous les 15 mètres environ (ces joints sont lisses, seulement enduits d'une peinture bitumineuse), ni aucun drainage particulier à la base des joints.

Des "trous de décompression" traversent le radier de part en part, sous forme de forages de $48 \mathrm{~mm}$ de diamètre à la maille de 3 à 5 mètres. Ils sont disposés en files, dans le sens amont-aval et raccordés au contact de la fondation, par des cordons drainants, indépendants les uns des autres et qui ne débouchent nulle part.

Une autre particularité est le joint de construction transversal qui sépare le tapis du pied aval du barrage. Il débouche dans la courbe de raccordement du déversoir au tapis, où le terme cinétique de l'écoulement est complété par la force centrifuge, et se présente incliné vers l'amont en forme de véritable prise de pression !

On sait aujourd'hui que de telles dispositions sont formellement à éviter. On lutte systématiquement contre le risque d'injection accidentelle de la pression cinétique dans les joints des coursiers d'évacuateurs, en combinant étanchéité et drainage dans ces joints. On veille à éviter la possibilité de mouvements relatifs des dalles secouées par un ressaut. On proscrit aussi l'introduction à la sous-face d'un radier des pressions pulsatoires du ressaut, d'autant plus que cela peut conduire à des combinaisons défavorables avec localement surpression en dessous et dépression au-dessus.

\subsection{Remèdes immédiats}

Avant même d'entreprendre sur modèle réduit hydraulique les essais décrits ci-après, destinés à vérifier ou réviser la disposition générale du déversoir, un premier ensemble de travaux de consolidation a été décidé.

Il s'agit essentiellement de mettre en place une série d'ancrages passifs pour lester le tapis et, aux endroits les plus exposés, empêcher son soulèvement par la pression cinétique (Fig. 6).

Toutes les communications directes entre les deux faces du tapis seront soigneusement obturées : on bouchera les perforations qui traversent les dalles, et l'on tentera de fermer les joints. On veillera surtout à éliminer tous les décrochements susceptibles de recueillir la pression cinétique dans ces joints.

Le détail des meulages ou réparations de joints sera précisé après l'inspection du tapis, noyé sous $3,5 \mathrm{~m}$ d'eau et dont la mise à sec représente, en elle-même, un gros effort.

Une série de drains subhorizontaux complète le dispositif : forés depuis la galerie de contrôle du barrage, ils passent dans le rocher de fondation juste sous le pied aval du barrage et sous le joint incliné de raccordement du tapis.

Les ancrages sont dimensionnés pour équilibrer : - le déficit de poids qui apparaît lorsque le ressaut est

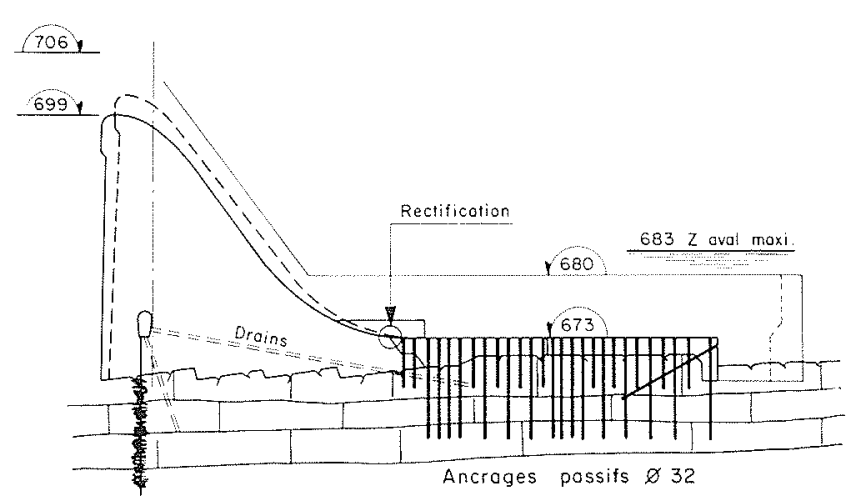

Figure 6 - Confortement primaire. 
chassé (la sous-face du tapis étant au niveau piézométrique aval),

- un minimum d'effets pulsatoires.

On prévoit, en zones courantes, un ancrage en acier $\phi 32$ TOR tous les $1,5 \times 1,5=2,25 \mathrm{~m}^{2}$, d'une longueur de 6 et 12 mètres alternativement, planté verticalement. Les aciers travaillent normalement à $26 \mathrm{~kg} / \mathrm{mm}^{2}$, pour reprendre $10 \%$ du terme $V^{2} / 2 \mathrm{~g}$ en plus du déficit de poids. Ils approcheraient de leur limite élastique pour plus de $40 \%$ du terme cinétique.

Sur l'amont des dalles, zones les plus exposées à l'introduction de la pression cinétique, les ancrages seront renforcés pour accroître la force de couture disponible au contact béton-rocher. On veut en effet éviter l'ouverture de ce contact par des pointes de pulsations liées au ressaut, pointes dont on sait qu'elles peuvent atteindre $40 \%$ du terme $V^{2} / 2 \mathrm{~g}$ au lieu de 10 à $15 \%$ en moyenne.

Le renforcement des ancrages à l'amont devrait suffire pour combattre les effets pernicieux de l'introduction de la pression cinétique dans les joints dépourvus de lames d'étanchéité : le strict contrôle de la continuité du parement hydraulique sur les joints empêchera en effet à cette pression d'être à la fois complète et généralisée.

$\mathrm{Au}$ total, 20000 mètres d'ancrages vont être ainsi mis en œuvre à Djorf-Torba dans les dix huit mois qui viennent.

\section{Etude de restructuration}

\subsection{Objet des études effectuées sur modèle réduit hydraulique}

Un modèle réduit hydraulique a été mis en oeuvre pour :

- préciser les limites de stabilité du ressaut sur le tapis, compte tenu des possibilités d'alimentation du centre par les côtés (que l'analyse théorique bidimensionnelle ne peut pas prendre en compte),

- apprécier les risques d'affoullements en l'état actuel des ouvrages, compte tenu de l'absence de seuil rocheux aval,

- rechercher et mettre au point des dispositions satisfaisantes pour la restitution des eaux dans le Guir.

Dès le départ, on s'était imposé de conserver le tapis à sa cote actuelle : il serait inconcevable en effet de l'abaisser alors que le barrage est en exploitation et risque de déverser pendant les travaux.

Plusieurs voies de recherche avaient été envisagées a priori sur cette base :

- déflecteur type tremplin à l'extrémité aval du tapis, accompagné d'un parafouille approfondi,

- ou contre-barrage plus à l'aval (reconstituant le seuil rocheux introuvable, mais nécessitant probablement de prolonger le tapis jusqu'à ce seuil),

- ou encore tremplins-aérateurs de type "splitter" sur le coursier incliné,

- sinon même saut de ski au pied même du coursier en amont du tapis.
Une préétude théorique montrait l'intérêt probable d'un seuil franc de quelques mètres au bord aval du tapis, sans renseigner cependant sur les affouillements à en attendre.

\subsection{Procédure suivie pour les essais}

Le modèle a été construit au L.C.H.F. à l'échelle $1 / 50$, en intégrant $200 \mathrm{~m}$ de retenue à l'amont et $500 \mathrm{~m}$ de vallée à l'aval. La maquette, haute de $70 \mathrm{~cm}$ et large de $2 \mathrm{~m}$, a été réalisée avec des formes guides en plastique et un remplissage en béton.

Selon les essais, le lit de l'Oued était fixe (au niveau présumé du rocher sous les alluvions), ou mobile (sable représentant les alluvions, reposant sur des graviers concassés simulant le fond rocheux délitable). Les alluvions étant chassées dès que le débit atteint $800 \mathrm{~m}^{3} / \mathrm{s}$, on ne les a représentées que dans les premiers essais. Le rocher était supposé se déliter en blocs de 100 à $500 \mathrm{~kg}$ dans les premiers essais d'érosion, et même de 4 à $40 \mathrm{~kg}$ pour les ultimes essais de validation de la solution retenue. On a pris soin de placer l'ensemble du modèle assez haut au-dessus du plancher du hall d'essai, de sorte que les affouillements n'ont jamais atteint le fond fixe.

Le niveau aval était réglé au droit de la section de mesure connue, située à $250 \mathrm{~m}$ de l'extrémité du tapis. On a systématiquement testé la sensibilité des écoulements observés à des modifications éventuelles de ce niveau.

Les contributions respectives du centre et des côtés variant rapidement avec le débit total, les essais ont été effectués sur une gamme complète de débits plutôt que sur le seul débit maximal.

Les essais ont été menés comme suit :

1. Etat actuel en lit fixe, détermination des lois de débit avec et sans ouverture des vidanges de fond.

2. Affouillements en l'état actuel.

3. Fonctionnement hydraulique en l'état actuel en lit à nouveau fixe (sous les alluvions), sensibilité au niveau aval, examen qualitatif des différents types de solutions envisagées.

4. Essais systématiques de différents seuils aval, en lit toujours fixe, puis mise au point de deux solutions: a) séparation complète des écoulements sur le tapis par deux murs bajoyers; centre traité par tremplin aval et côtés traités par seuil aval de deux mètres,

b) seuil aval continu de deux mètres à l'extrémité du tapis, complété par deux blocs déviateurs placés sur l'amont du tapis dans l'axe des passes centrales. Optimisation de la forme et de la disposition de ces blocs. 5. Retour en lit affouillable (rocher seul représenté), et essais d'érosion sur les deux solutions ainsi développées. Remarquable modicité des érosions. Passage à un gravier plus fin. Enlèvement des blocs déviateurs pour ne conserver que le seuil aval.

\subsection{Principales observations effectuées dans l'état actuel}

Le modèle réduit hydraulique constitue un moyen d'analyse irremplaçable, pour prendre en compte l'aspect tridimensionnel du ressaut sur le tapis, aspect qui résulte đu décalage des seuils. Le contraste entre les écoule- 


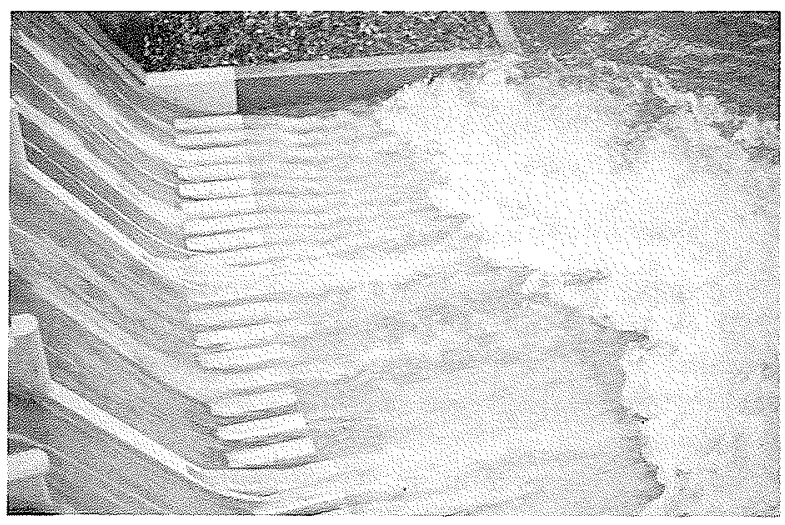

Figure 7 - Modèle réduit hydraulique dans l'état actuel : ressaut presque entièrement chassé du tapis pour $2000 \mathrm{~m}^{3} / \mathrm{s}$.

ments au centre et sur les côtés apparaît atténué par rapport à ce qu'indiquait l'analyse préalable bidimensionnelle : le ressaut présente une continuité remarquable sur toute la largeur du tapis, étant seulement plus reculé au centre que sur les côtés.

Le ressaut se détache assez vite des chute-blocs, en particulier au droit des passes centrales où il les quitte franchement dès que le débit dépasse $300 \mathrm{~m}^{3} / \mathrm{s}$. L'influence des vidanges de fond est assez marginale. Le ressaut quitte progressivement le tapis entre 2000 et 3000 $\mathrm{m}^{3} / \mathrm{s}$, plus ou moins tôt selon le niveau aval et toujours en commençant par la partie centrale. A $2000 \mathrm{~m}^{3} / \mathrm{s}$, le tiers amont du tapis est dégagé sur les côtés, et le ressaut est plus ou moins sorti du tapis au centre. A $3000 \mathrm{~m}^{3} / \mathrm{s}$, ne restent plus sur le tapis que les extrémités latérales du ressaut, encore accrochées aux murs bajoyers (Fig. 7).

Les vitesses de l'écoulement à l'aval du tapis dépasssent les $10 \mathrm{~m} / \mathrm{s}$ à $2000 \mathrm{~m}^{3} / \mathrm{s}$ et les $20 \mathrm{~m} / \mathrm{s}$ à $3000 \mathrm{~m}^{3} / \mathrm{s}$. Des érosions inacceptables résultent de ces conditions : la cote 668 de fondation du parafouille aval et des coins des bajoyers, $5 \mathrm{~m}$ sous le niveau du tapis, est assez rapidement atteinte de façon sporadique. Le déchaussement apparait général, à cette cote, pour un débit de 2500 $\mathrm{m}^{3} / \mathrm{s}$, et descend encore de 3 à $5 \mathrm{~m}$ en certains endroits pour $3000 \mathrm{~m}^{3} / \mathrm{s}$.

Ces résultats recoupent assez bien les indications du premier modèle réduit hydraulique, mis en œuvre à l'Institut Cerni de Belgrade en 1966 au début des travaux de construction du barrage.

On a vérifié que les érosions étaient sensiblement les mêmes en imposant un fort débit instantané $(2000$ $\mathrm{m}^{3} / \mathrm{s}$, ou en suivant sur le modèle une croissance du débit par paliers successifs de 20 heures ( $=3$ heures modèle).

Les essais d'érosion ont fait apparaître une "dune" de matériaux qui se déposent à l'aval de la fosse, et créent des conditions de niveau plutôt favorables pour le maintien du ressaut sur le tapis. Il n'est pas certain que le rocher, une fois érodé, ne se délite pas en plus petits blocs que ceux représentés sur le modèle ce qui abaisserait la dune. Il n'est pas certain non plus que le rocher soit aussi érodable que représenté sur le modèle. On a donc développé l'étude des écoulements sur le tapis, dans l'état actuel et pour la recherche de solutions, en revenant en lit fixe.

\subsection{Recherche de solutions}

Cette recherche a été menée avec un souci d'économie et de simplicité, ce qui a permis d'éviter:

- l'allongement du tapis,

- l'approfondissement du parafouille existant, auquel on s'attendait au départ, (ce qui est du plus grand intérêt au plan des travaux).

Compte tenu de la rareté des fonctionnements prévisibles, quelques risques de cavitation localisée ont été admis.

La recherche a été conduite au début en lit fixe, pour s'affranchir comme indiqué ci-avant du relèvement du niveau aval par la dune.

L'effet de seuils disposés à l'extrémité aval du tapis, plus ou moins hauts et de profils plus ou moins agressifs, a tout d'abord été examiné en détail. Le critère essentiel était le maintien du ressaut sur le tapis, les critères secondaires étant l'uniformité du ressaut et la minimisation du deuxième ressaut apparaissant en certains cas en aval du seuil. Le seuil optimal est apparu avoir une hauteur de 2 mètres (Fig. 8). Une forme simple, de section carrée, a été jugée plus aisée à réaliser que le profil en redan qui avait donné le meilleur résultat.

L'écoulement derrière ce seuil restant très violent, la solution a été développée dans deux directions contrastées (Fig. 9, 10, 11) entre lesquelles on se réservait de

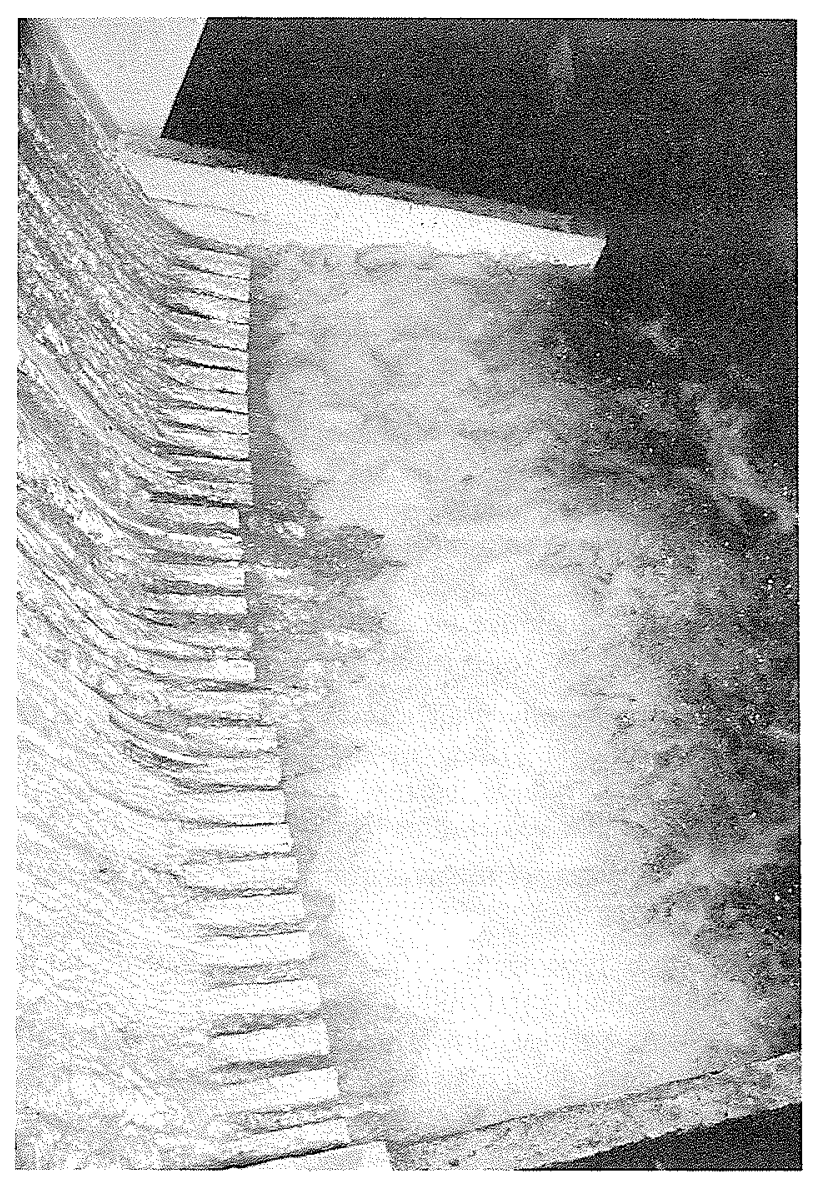

Figure 8 - Ressaut contenu sur le tapis pour $2000 \mathrm{~m}^{3} / \mathrm{s}$, avec le seuil aval de $2 \mathrm{~m}$ finalement retenu. 


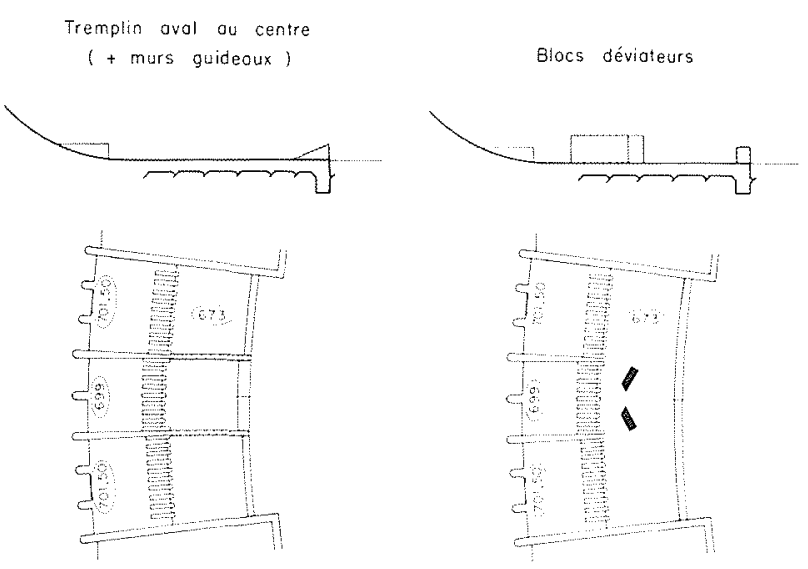

Figure 9 - Solutions testées:

a) avec tremplin aval au centre, et murs guideaux : b) avec blocs déviateurs.

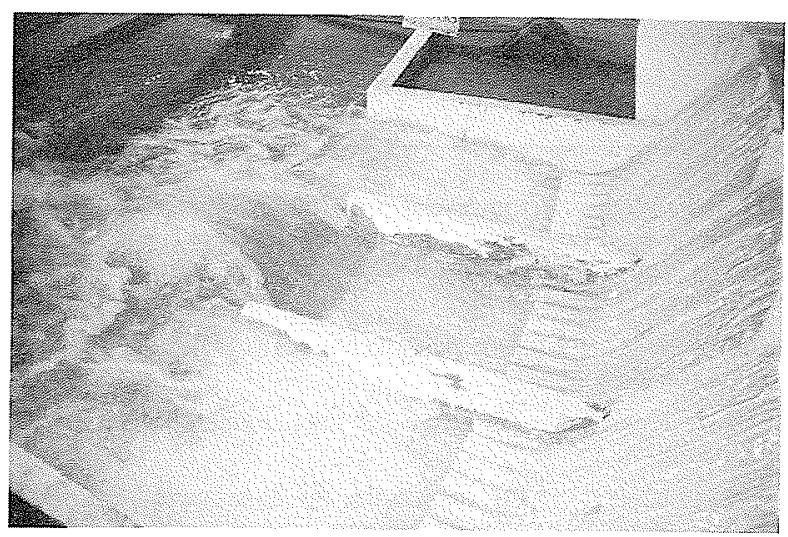

Figure 10 - Rejet de l'impact central par tremplin aval, avec murs séparatifs sur le tapis.

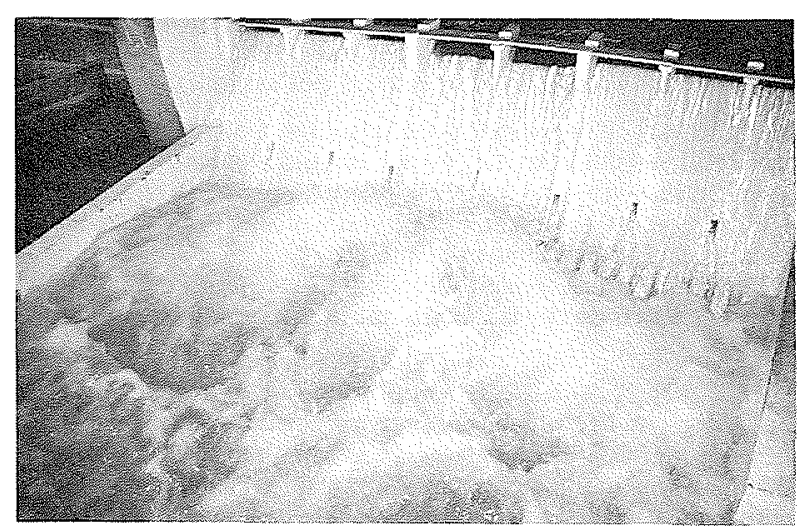

Figure 11 - Effet de blocs déviateurs, associés à un seuil continu aval.

trancher au vu des essais d'érosion :

- un rejet au loin de l'impact central par tremplin aval, ce qui imposait une séparation franche des écoulements avec des murs bajoyers intermédiaires édifiés sur le tapis; cette option était hydrauliquement très satisfaisante ma is assez lourde au niveau des travaux,
- le recours à des blocs déviateurs placés sur l'amont du tapis et destinés :

- à rejeter une partie des débits du centre vers les côtés, - à participer à la dissipation d'énergie sur le tapis, - et à lutter contre les courants de retour susceptibles de déchausser les coins des bajoyers.

Cette deuxième option a fait l'objet de développements intéressants : les formes et la disposition des blocs ont pu être ajustées pour localiser les impacts sur le tapis, et uniformiser le débit de sortie vers l'aval. Mais ce faisant, on relevait sensiblement les lignes d'eau le long des bajoyers latéraux, qu'il fallait déjà surélever de plusieurs mètres avec le seuil aval seul.

Le retour en lit affouillable a montré l'excellence des deux solutions développées: le rocher au pied des ouvrages n'était creusé qu'aux débits extrêmes, exclusivement aux coins aval des murs bajoyers. Mais le prix à payer paraissait lourd, compte tenu de la relative rareté des fonctionnements du déversoir. Un minimum d'allègements devait pouvoir être consenti.

\subsection{Choix final}

C'est finalement la solution du seuil aval continu seul, sans blocs déviateurs, qui a été retenue. Elle est accompagnée d'une surélévation des bajoyers existants, modulée d'amont en aval (Fig. 12), ainsi que d'une suré. lévation des murs en retour destinée à couper les alimen. tations par l'aval qui pourraient trop charger, dans le mauvais sens, des bajoyers dont l'embase est imposée.

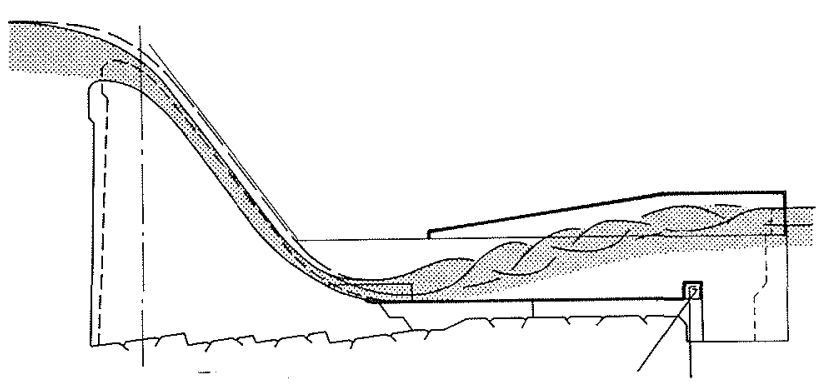

Figure 12 - Restructuration finale et lignes d'eau extrêmes.

Les essais d'érosion ont été particulièrement développés pour valider la solution ainsi retenue, en considérant :

- la gamme progressivement croissante des débits, suivie d'un retour à $800 \mathrm{~m}^{3} / \mathrm{s}$,

- un niveau aval réglé volontairement bas,

- un rocher supposé sensiblement plus délitable que dans les autres essais.

Des érosions se produisent contre le bord aval du tapis, sans que leur profondeur ne devienne excessive : cote 671 à 672 au pire, soit encore 3 à $4 \mathrm{~m}$ au-dessus du fond du parafouille.

Les déchaussements maximaux apparaissent contre les coins aval des murs bajoyers (cote 669 pour 3500 $\mathrm{m}^{3} / \mathrm{s}$ ). On s'en protègera en renforçant l'ancrage et le monolithisme des coins bajoyers/murs en retour à l'occasion de la surélévation, en ancrant la base des bajoyers et en disposant des réserves de gros enrochements contre les nurs en retour. 


\section{Conclusion}

Le modèle réduit hydraulique a constitué un outil irremplaçable pour l'étude et l'amélioration de la restitution du déversoir de crues de Djorf-Torba, étude rendue nécessaire par une révision en hausse des estimations hydrologiques et des risques de déversements.

Cet évacuateur de crues n'a pas encore fonctionné véritablement 14 ans après son achèvement. Il est typique des grands évacuateurs non vannés d'Afrique du Nord qui ne voient presque jamais passer leur crue de projet. Mais il suffit d'une fois. . .

L'ouvrage, en son état actuel, est exposé à un risque inacceptable de déchaussements, et muni de bajoyers latéraux insuffisants. Outre l'indispensable surélévation de ces bajoyers, la restructuration pourra se limiter à l'édification d'un simple seuil de $2 \mathrm{~m}$ au bord aval du tapis existant, lui-même systématiquement renforcé par une série d'ancrages passifs plantés verticalement dans le banc calcaire sousjacent. Le seuil sera naturellement fixé par des ancrages analogues.

Les travaux, qui viennent de commencer, seront aussi l'occasion de rectifier diverses dispositions dangereuses pour la stabilité du tapis : suppression des communications hydrauliques entre ses deux faces, et suppression des angles défavorables sur les joints de construction.

Ainsi auront été mis à profit les acquis de la technique hydraulique pour assurer la pérennité de ce grand ouvrage.
Exposé de M. GOGUEL à la préparation duquel ont également collaboré MM. BELOUNI et SAHRAOUI, du Ministère de l'Hydraulique de l'Algérie et M. BOULOC du Laboratoire Central d'Hydraulique de France, à Maison-Alfort.

M. le Président. - Je remercie M. GoGUEL de son exposé très intéressant qui montre le ratrappage de certaines erreurs imputables, pour une grande part, à une insuffisance de données hydrologiques.

J'ouvre la discussion en posant une question à M. GOGUEL : Une des figures projetées montre un drain partant de la galerie du barrage et s'enfonçant sous la partie amont du tapis; le drainage actuel semblant insuffisant, quelle est la solution préconisée pour le rendre plus efficace?

M. GOGUEL. - Ces drains sub-horizontaux qui partent de la galerie du barrage constituent une mesure immédiate prise pour recouper les fissures sous le joint barrage-tapis et éviter l'injection de pressions excessives sous le pied aval du barrage-poids.

Une galerie de drainage passant sous le tapis a été envisagée, mais son exécution s'avérait extrêmement difficile et l'efficacité $\mathrm{du}$ drainage vis-à-vis des surpressions pulsatoires, en milieu saturé, était douteuse. Le réseau de drainage aurait été, en effet, saturé par le niveau aval, un système d'épuisement par pompage étant impensable.

M. MEILLAND. - Pourquoi n'a-t-on pas réalisé un drainage derrière les blocs de pied du coursier, au moyen de trous verticaux de décompression?

M. GOGUEL. - Il serait tentant effectivement de mettre en place des drains débouchant aux endroits en dépression. Mais n'étant pas sûr qu'il y ait dépression dans tous les cas, c'est-àdire pour toute la plage des débits et donc des positions du ressaut au cours d'un déversement, à l'aval des chute-blocs et que ceux-ci auraient tenu, nous avons préféré écarter une telle solution.

M. GRUAT. - Quelle est l'utilité des chute-blocs, solution assez souvent adoptée, notamment aux Etats-Unis? Notre Laboratoire de Toulouse les a essayés, mais, dans la majorité des cas, les a supprimés car ils ne sont pas favorables à la stabilité du ressaut.

M. GOGUEL. - Lorsque nous sommes intervenus sur ce barrage, les chute-blocs étaient déjà en place puisque l'ouvrage est construit depuis près de dix ans.

M. PELLERAY. - Les trous existant dans les dalies ont-ils été bouchés?
M. GOGUEL. - Nous avons prévu de les obturer après avoir examiné le ty pe de communication existant entre ces trous. Pour l'instant, cet examen n'a pu être effectué en raison de la présence de vase sur le tapis.

M. ALAM. - N'est-ce pas grâce à ces trous que le tapis n'a pas été soufflé ?

M. le Président. - Si ces trous sont bouchés, il y aura des sous-pressions.

M. GOGUEL - Ces trous dits de décompression doivent éviter au tapis d'être soufflé lorsque le bassin, est mis à sec, le niveau statique aval de l'oued pouvant s'établir sous le tapis. Lorsque les trous seront bouchés, il faudra certes avoir des ancrages pour tenir le tapis. Mais relativement peu.

Par contre, lorsque le ressaut se déplace sur le tapis, ces trous peuvent provoquer des surpressions et les transmettre ailleurs. Dans certains cas, de telles surpressions risquent de se combiner, localement, avec des dépressions résultant du ressaut, ce qui peut entraîner des sollicitations, d'ailleurs très complexes, du tapis.

M. $A L A M$. Il me semble que la solution retenue ne soit pas tout à fait satisfaisante. Le ressaut est relativement haut à l'aval et peut transmettre des pressions jusqu'au début de la dalle dont la stabilité n'est pas assurée à ce moment-là. Peut-on vraiment compter sur la bonne tenue des ancrages?

Personnellement, j'aurai préféré d'autres solutions plutôt que d'avoir un ressaut hydraulique presque complètement chassé du bassin.

M. GOGUEL - Dans la solution définitive de confortement consistant à mettre en place un seuil continu, en béton armé, ancré sur le bord aval du tapis, le ressaut sera beaucoup mieux stabilisé et maintenu sur le tapis, comparativement à la solution actuelle.

Les dispositions de confortement recommandées sur l'ouvrage sont destinées d'abord à parer au plus pressé, c'est-à-dire à ancrer le tapis pour éviter qu'il ne soit soufflé - dans une deuxième phase, on essayera d'améliorer les conditions de restitution pour éviter que le tapis ne soit déchaussé.

M. ALAM. - N'est-ii pas possible de réaliser un contrebarrage à l'aval dans le lit même de la rivière pour noyer le ressaut?

M. GOGUEL. - Le ressaut se produira toujours sur le tapis qui sera ainsi soumis à des chargements pulsatoires. Il peut y avoir combinaison défavorable de surpressions injectées à sa sous-face et de dépressions à sa surface; seul un traitement de 
peau pourrait éliminer cette combinaison. Le contre-barrage est une solution qui a été envisagée, mais elle présente des travaux d'emprise considérable, avec la nécessité, sans doute, de tapisser le fond rocheux derrière le tapis. De plus, il faut signaler qu'il est très difficile de mettre à sec le fond rocheux du lit de la rivière pour y travailler, une galerie de drainage évacuant en permanence dans l'oued un débit de 300 à $500 \mathrm{l} / \mathrm{s}$. Donc, plus le chantier sera limité, plus les travaux seront exécutés rapidement.

M. SELMI. - Quelle est la hauteur retenue pour le seuil à l'aval du barrage ? Est-ce que plusieurs hauteurs ont été étudiées sur le modèle?
M. GoGUEL. - Oui, des hauteurs de 2,3 et $4 \mathrm{~m}$ ont été testées sur modèle et c'est la hauteur de $2 \mathrm{~m}$ qui a été retenue pour éviter la remise en vitesse et le ressaut secondaire qui se développe à l'aval d'un seuil quand celui-ci est trop haut.

M. BOULOC. - En réponse à la question posée par $M$. SELMI, je voudrais également préciser que la hauteur de $2 \mathrm{~m}$ adoptée est la hauteur minimale maintenant le ressaut au pied même du barrage. Un seuil plus haut aurait entrainé une remise en vitesse, et créé une autre chute sur ce deuxième barrage nécessitant une protection de l'oued à l'aval.

\section{Abstract \\ Restructuring of the spillway of the Djorf-Torba dam}

There is a reason Djorf-Torba dam, built in 1968 on the Guir, impounds the largest man-made reservoir in Algeria, and contols a sub-Saharan catchment of $22000 \mathrm{~km}^{2}$. It is a concrete gravity structure, $35 \mathrm{~m}$ in height, with a 650 million $\mathrm{m}^{3}$ flood attenuation capacity above the 250 million $\mathrm{m}^{3}$ operating capacity, providing a considerable degree of flood control.

Records acquired since the construction of the dam however, have led to an upward increase in the estimate of the flood risk, with a greater likelihood of spilling $1000-3500 \mathrm{~m} 3 / \mathrm{s}$. The essential part of the dam has tiered sills, and behind the dam there is a $100 \mathrm{~m}$ wide apron.

A proper hydraulic-jump stilling pool would have been set 5 to $10 \mathrm{~m}$ lower, and would probably have been much stronger. The $2 \mathrm{~m}$ thick concrete apron has no reinforcement and is not pinned down to the rock, and there are no seals in the construction joints. One of the joints at the upstream end even seems to be dangerous because it slopes towards upstream, so that it might channel the water pressure under the slab.

The first phase of the remedial works consisted of pinning the apron to the limestone foundation rock by means of unstressed vertical rods, to provide extra weight and combat uplift forces from the velocity hear or turbulence in the hydraulicjump. This consists of $32 \mathrm{~mm}$ TOR. bar on $1.5 \mathrm{~m}$ centres both ways, i.e. 1 per $2.25 \mathrm{~m}^{2}$. They are alternately $6 \mathrm{~m}$ to $12 \mathrm{~m}$ long, and concentrated at the upstream edges of the slabs. This makes a total of $20000 \mathrm{~m}$ of anchor, to be installed in the next 18 months.

A hydraulic scale model was built to determine the stability limits of the hydraulic-jump on the apron, evaluate the underscour risk, and find the best means of channelling water back into the river. From the outset, it was decided to keep the apron at its present level, because the dam is operational and spillage may occur at any time. The 1/50 th scale model, built by the Laboratoire Central d'Hydraulique de France was invaluable in that it was able to handle the three-dimensional features of the jump caused by the tiered central and side sills. It was also an excellent tool in producing a design that would prevent the slabs lifting off the foundation.

The main steps in the test programme are described. After a few moths testing, it was clear that it would not be necessary to lengthen the apron or deepen the existing cut-off. But in its present condition, there is an unacceptable risk of the floor slabs loosening. The solution will be to build a plain continuous reinforced concrete wier $2 \mathrm{~m}$ high at the downstream edge, and raise the existing training walls.

Construction work has just commenced, and will be a new step forward in modern hydraulic design. 